\title{
Legume consumption and severe depressed mood, the modifying roles of gender and menopausal status
}

\author{
Yanfeng $\mathrm{Li}^{1}$, Qi Dai ${ }^{2}$, Stuart $\mathrm{H}$ Tedders ${ }^{3}$, Cassandra Arroyo ${ }^{3}$ and Jian Zhang ${ }^{3, *}$ \\ 'Department of Epidemiology and Biostatistics, Arnold School of Public Health, University of South Carolina, \\ Columbia, SC, USA: ${ }^{2}$ Department of Medicine and Vanderbilt-Ingram Cancer Center, Vanderbilt University, \\ Nashville, TN, USA: ${ }^{3}$ Jiann-Ping Hsu College of Public Health, Georgia Southern University, PO Box 8015, \\ Statesboro, GA 30460-8015, USA
}

Submitted 10 February 2009: Accepted 12 October 2009: First published online 26 January 2010

\begin{abstract}
Objective: Legumes have been recommended as staple foods in the anticipation of disease prevention. However, the scientific evidence of their benefits, particularly on mental well-being, remains preliminary. We longitudinally assessed the association between legume consumption and the risk of severe depressed mood (SDM) among a national cohort.

Design: The study included adults aged 25-74 years who were examined in 1971-1975 as a part of the National Health and Nutrition Examination Survey. Legume consumption at baseline was obtained from a 3-month FFQ and categorised as infrequent ( $<1$ time/week), moderate (1-2 times/week) and frequent $(\geq 3$ times/week). SDM was defined as Center for Epidemiologic Studies Depression Scale score $\geq 22$ or taking anti-depression medication after an average of $10 \cdot 6$ years of follow-up (from $8 \cdot 0$ to $12 \cdot 5$ years).

Results: Among women, the proportion of individuals with SDM was $17 \cdot 75 \%$. For premenopausal women ( $n$ 1778), a significant linear trend of deleterious effect from legume consumption was observed ( $P$ for trend $=0 \cdot 0148$ ). The relative risks (RR) for infrequent, moderate and frequent consumptions were 1 (reference), $1.24(95 \% \mathrm{CI}=0.91,1.70)$ and $1.75(1.12,2.75)$, respectively. However, moderate consumption showed a significant protective effect $(\mathrm{RR}=$ $0 \cdot 52(0 \cdot 27,1 \cdot 00))$ among women undergoing the menopausal transition $(n 454)$. No association was obtained from either postmenopausal women $(n 601)$ or men ( $n$ 2036).

Conclusions: These findings suggest that gender and menopausal status were effect modifiers of the association between legume consumption and SDM. Detrimental effects of frequent consumption of legumes may exist among premenopausal women; moderate consumption, however, may protect perimenopausal women against SDM.
\end{abstract}

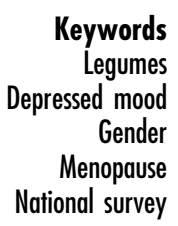

Due to their unique nutritional values, legume seeds have been recommended as a staple food by various health organisations in an anticipation to guard against CVD ${ }^{(1)}$, cancers $^{(2)}$ and type 2 diabetes $^{(3)}$. Legumes are low in fat and rich in protein, fibre, minerals and vitamins. Frequent intake of legumes may lower blood cholesterol concentration and reduce the risk of $\mathrm{CHD}^{(4)}$. The low glycaemic index of legumes also contributes to improved blood glucose control ${ }^{(5)}$. The presence of certain amounts of non-nutritional factors, such as isoflavones, has been linked with a number of health-promoting properties. Specifically, these factors are considered naturally occurring bioactive substances that may play an important role in the prevention of CVD, as well as some types of cancer ${ }^{(6)}$.
In spite of a large body of information supporting the health benefits of legumes, a panel of experts that reviewed the evidence of an association concluded that there was a lack of definitive scientific information on the link between legumes and human health ${ }^{(7)}$. The panel recommended in its 2005 report that 'Basic research is required to demonstrate, using in vitro experiments, suitable animal models, and, in the clinics, the true health benefit of grain legumes, namely on cancer, CVD, diabetes and obesity prevention or treatment ${ }^{(8)}$. Following the release of the updated results of the Women's Health Initiative ${ }^{(9)}$, research on the nutritional value of legumes to human health is of increasing interest given that many women are reluctant to use exogenous hormones for 
symptomatic treatment and are turning to botanicals and dietary supplement products for relief. However, the rapidly expanding literature has suggested that the highly celebrated health benefits of soya, the main type of legumes consumed, are not entirely merited ${ }^{(10-13)}$. In addition to protein, fibre, minerals, vitamins and isoflavones, legumes contain a large number of unhealthy components, including phytates. Phytates could block the body's uptake of essential minerals like magnesium, calcium, iron and especially Zinc $^{(14)}$. The primary claims about legumes' health benefit come from the observation that Asian women, who eat legume produce much more often than their American counterparts, suffer far fewer cases of breast cancer than American women. The observation, however, did not take into account that Asian women eat a diet consisting of more natural products, greater amounts of vegetables and more fish, dramatically different from their American counterparts $^{(15)}$. Asian diets are also lower in chemicals and toxins due to far fewer processed (canned, jarred, pickled and frozen) foods. All these facts make the extensive investigation on health effects of legume consumption urgently necessary. It is also noted that the effects of legume consumption on psychiatric well-being have not been investigated. Therefore, we took advantage of the prolonged follow-up experience of participants in the National Health and Nutrition Examination Study I Epidemiologic Follow-Up Study (NHEFS) to examine the relationship of legume consumption on the risk of depressed mood in a nationally representative sample of the US men and women.

\section{Data and methods}

\section{Study population}

Detailed descriptions of the NHEFS have been published elsewhere $^{(16-20)}$. In brief, the NHEFS was a cohort study of adults who participated in the first National Health and
Nutrition Examination Survey (NHANES I) from 1971 to 1975 as the baseline survey. From 1982 to 1984, a followup survey was conducted and, of the original NHEFS cohort ( $n$ 14407), 14\% ( $n$ 2022) were deceased, $7 \%$ ( $n$ 1024) were lost to follow-up and 79\% ( $n$ 11361) of the participants were successfully traced and interviewed. The current study included the NHEFS participants who remained alive and who completed information on depressive symptoms assessed in 1982-1984 ( $n$ 9517). In our study, participants were excluded if $24 \mathrm{~h}$ dietary recall was recorded as unsatisfactory $(n 70)^{(21)}$, data on legume consumption were missing from the FFQ ( $n$ 2245), information on alcohol and cigarette consumption was unavailable ( $n$ 1776), or data on socio-economic status (SES), including income level, education attainment, marital status and type of residential area, were not available ( $n$ 200). A total of 163 participants were excluded due to an unavailability of BMI $\left(\mathrm{kg} / \mathrm{m}^{2}\right)$, serum total cholesterol at the baseline, self-evaluated health status and the history of major physical diseases at the followup. Additionally, 194 women were excluded because of 'too early' (before the age of 43 years) or 'too late' (after the age of 56 years) cessation of menses. Thus, a total of 4869 subjects were available for this analysis (Fig. 1).

\section{Severe depressed mood}

Depressed mood was assessed in the 1982-1984 follow-up using the Center for Epidemiological Studies Depression Scale (CES-D) questionnaire. The CES-D questionnaire was developed for epidemiological surveys of the general population to measure depressive feelings and behaviours during the past week ${ }^{(22)}$. It consists of twenty descriptive statements of depressed mood, feelings of worthlessness, hopelessness and loneliness, loss of appetite, sleep disturbances, problems of concentration and psychomotor retardation. The survey was scored on a standard 4-point scale from 0 to 3, and participants were asked to rate each

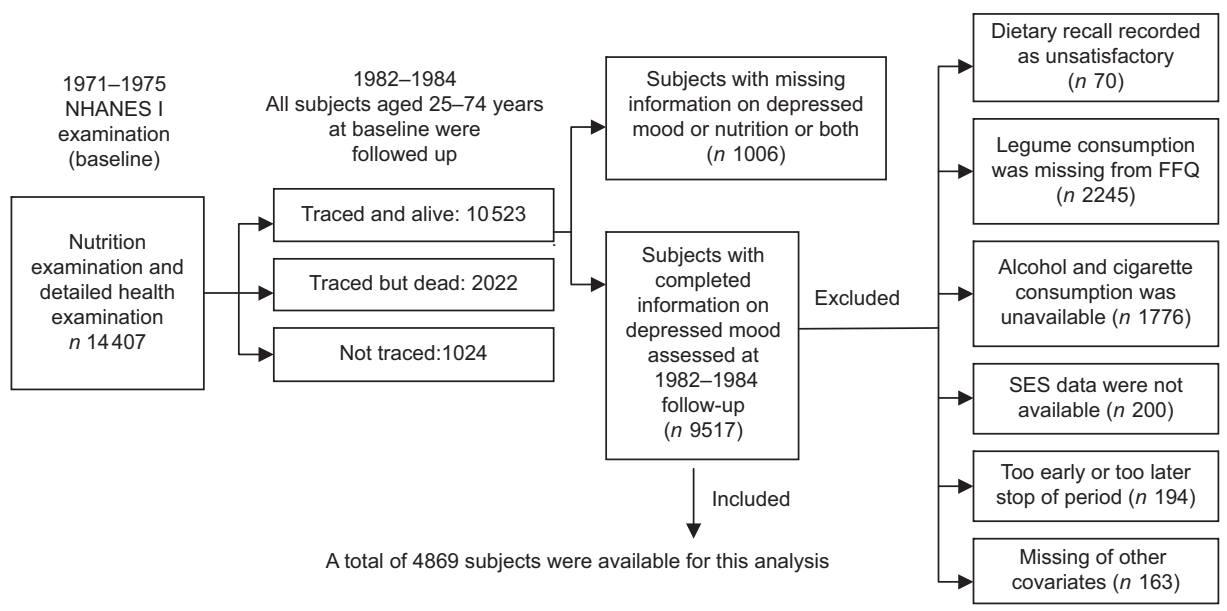

Fig. 1 Flow chart of the study population included 
item according to the frequency experienced in the past week. The twenty items had a potential range of $0-60$, with the higher scores representing responses in the depressed range. Only persons who answered all twenty items on the questionnaire were included in the current analysis. Those with a total score of $\geq 16$ on the CES-D, which corresponds approximately to the 80th percentile score, were considered to have moderate depressed mood or severe depressed mood (SDM). In the preliminary analyses, we observed that the CES-D $\geq 16$ threshold was not able to identify distinct levels of severity of the symptoms, thus not providing adequate statistical power to detect the association. On the basis of this preliminary analysis, participants were categorised as having SDM, if the CES-D score was $\geq 22$. In addition, individuals taking anti-depression medication when the follow-up interview was conducted were also categorised as having SDM.

\section{Legume consumption}

A 3-month FFQ was administered at the baseline survey (1971-1975) by trained interviewers, usually registered dietitians. The questionnaire covered the 3 months before the interview and assessed usual consumption patterns excluding periods of illness or dieting. Data were collected on eighteen groups and included daily and/or weekly consumption for all regular meals and snacks ingested every day including the weekends and holidays. The interviews were conducted in specially designed mobile examination units. On-site evaluations, review of questionnaires and taped interviews were conducted as part of the data quality control. Only questionnaires coded as satisfactorily completed by interviewers were used in this analysis. The questionnaire on the primary food item used in this investigation asked participants how often 'dry beans and peas like pinto beans, red beans, black-eye peas, peanuts and peanut butter' were usually consumed in the past 3 months, excluding periods of illness or dieting. To identify distinct levels of consumption and to provide adequate numbers in each category, we categorised legume consumption into three levels: (i) infrequent (never or less than once a week), (ii) moderate (once or twice a week) and (iii) frequent (three times or more a week).

\section{Major confounders and effect modifiers}

Gender is a well-established risk factor for mood dis$\operatorname{order}^{(23)}$, and dietary patterns are largely genderspecific $^{(24)}$. Therefore, we performed separate analyses for men and women. Preliminary analyses indicated that menopausal status was an effect modifier; we further stratified women into three groups using both age at baseline interview and their answers to the question 'Have they (periods) entirely stopped?' Women aged 25-43 years whose periods had not stopped were classified as premenopausal. The classification group termed perimenopausal included women aged $44-55$ years undergoing the menopausal transition regardless of the answer to the question above. Women aged 56 years or older whose periods had entirely stopped were grouped as postmenopausal. Women aged 43 years or younger, but with periods entirely stopped ( $n$ 190), and women older than 56 years, but with periods that had not yet stopped ( $n$ 4), were excluded from the current analysis.

\section{Otber covariates}

Covariates included SES, behavioural and dietary characteristics assessed at the baseline, self-evaluated health status and a history of major medical illnesses assessed at the follow-up survey. More than $90 \%$ of the subjects were White, so ethnicity was coded as Whites and others. Educational attainment at baseline was measured as the highest completed grade of school and categorised as four levels. Marital status was dichotomised as either married or others. Poverty status was defined by the ratio of family income to the federal poverty line threshold established annually by the US Bureau of the Census. Other SES variables included employment status (employed and unemployed including housekeeping), occupation (farm and non-farm) and type of residence area (urban, suburban and rural).

The category for alcohol consumption separated heavy (every/just about every day) and moderate drinkers (2-3 times a week) from less regular drinkers or nondrinkers. Information on cigarette smoking status at baseline was derived from responses to questions on lifetime smoking history obtained at follow-up interviews between 1982 and 1984 or later. The participants were classified as 'have smoked more than 100 cigarettes in lifetime', otherwise as 'not'. At the follow-up survey, the respondents were asked, 'Has a doctor ever told you that you had any of the following conditions?' This was followed by a list of thirty-nine chronic and acute conditions, some described with vernacular expressions. The most frequently reported conditions included arthritis, fractures, bladder infections and anaemia. We selected the conditions of cancer, stroke, heart attack and diabetes as potential confounders. A history of food allergy was also included due to its relevance with soyabean consumption. A self-evaluated health status with categories of excellent, very good, good, fair and poor was recoded as 'excellent' 'very good or good' and 'poor or fair' to reduce the likelihood of overspecification of regression models. The $24 \mathrm{~h}$ dietary recall, administered by the same trained staff before beginning the frequency questionnaire, provided such information as specific food items and their quantities ingested for all regular meals, between meal foods or snacks consumed on the day, midnight to midnight, preceding the interview for each sample person interviewed. All food ingested during the $24 \mathrm{~h}$ period was then reduced by a computer program to standard units of measure for actual dietary intake, including SFA, total 
cholesterol and total dietary energy. We included these variables as the surrogates to control for the potential confounding from dietary pattern. Taking dietary supplement was also assessed in the $24 \mathrm{~h}$ dietary recall, and was included as a covariate (yes/no).

\section{Statistical methods}

The NHANES I design involved stratification, several levels of clustering and special weighting of selected age and race groups; thus the SAS-callable SUDAAN software version 9.0 (Research Triangle Institute, Research Triangle Park, NC, USA) accounting for NHANES sampling methods was conventionally utilised. Hazard ratios derived from Cox proportional hazard regression would be preferable given the cohort design of the current study. However, we present the relative risks (RR) obtained from a typical logistical regression due to the following considerations. The CES-D scale was designed to identify individuals with current depressed mood, and was not able to measure past episodes; therefore, it is impossible to pinpoint the first date of onset after the baseline survey. Hence, we have to assume that the date of follow-up survey as the onset date of the current depressive episodes, if SDM presented. Consequently, the follow-up durations were relatively unified for the entire cohort regardless of the depression status, with an average being 10.6 years (ranging from 8.0 to 12.5 years). The estimates derived from the logistic regression in the preliminary analyses were almost identical to those obtained from the Cox proportional hazard analyses. Therefore, we presented the estimates from logistic regression only. We did not use the -2 log-likelihood test to simplify regression models; instead, all potential confounders were retained in the multivariable models.

\section{Results}

The proportion of individuals with SDM was significantly higher among women than among men. A total of 526 women and 236 men were identified as suffering SDM, the corresponding proportions were $17 \cdot 75(\mathrm{sE}=1 \cdot 01) \%$ and $11.69(0.92) \%$, respectively (Table 1$)$. Men and women shared several risks of SDM, such as living below the poverty line and low educational attainment. Cigarette smoking and poor self-evaluated health status was associated with SDM for both genders. However, women had their own set of risk factors of SDM including low dietary energy, low dietary intakes of SFA and cholesterol.

The proportion of men eating legumes frequently was significantly higher than that of women, $27 \cdot 22 v \cdot 16 \cdot 47 \%$. When the study population was further stratified by the levels of legume consumption within each gender (Table 2), the proportion of women with SDM was significantly higher among frequent legume consumers than among infrequent consumers, $23 \cdot 31 v \cdot 17 \cdot 09 \%(P<0 \cdot 01)$, but no such difference was observed in men. For both genders, the frequent legume consumers were generally younger, less educated and more likely to live below poverty. It was also noted that within each gender, compared with infrequent legume consumers, the frequent consumers had a significantly lower level of serum total cholesterol among women, and a significantly lower proportion of obesity among men.

Neither crude nor adjusted RR show a significant association between legume consumption and the risk of SDM among the 2036 men (Table 3). The adjusted RR were 1 (reference), $1.09(95 \% \mathrm{CI}=0.68,1.75)$ and $0.72(0.43,1 \cdot 19)$, respectively, for men with infrequent, moderate and frequent legume consumptions. Among women, however, a non-monotonic association was observed. A linear trend was shown among premenopausal women. The proportions of women with SDM were $14 \cdot 24,17 \cdot 82$ and $24 \cdot 38 \%$ for infrequent, moderate and frequent consumptions, respectively $(P=0.0019$ for linear trend). A U-shaped association was obtained among perimenopausal women $(P=0.0517$ for quadratic trend). The proportions of perimenopausal women with SDM were $19 \cdot 32,11 \cdot 38$ and $20.51 \%$ for infrequent, moderate and frequent consumptions, respectively, indicating a protective effect from moderate legume consumption. Adjustment for SES, other dietary factors (i.e. frequency of intake of fruits and vegetables, and intake of SFA) and biological factors (i.e. food allergy, BMI and cholesterol) resulted in minimal changes in the estimates. No significant association was observed among postmenopausal women; however, a non-significant quadratic trend appeared, indicating the protective effect from moderate consumption of legume continues after perimenopause, but less is significant.

\section{Discussion}

We observed a gender-age-dependent association between legume consumption and risk of SDM. Among premenopausal women, consumption of legumes was associated with an increased risk of SDM in a dose-response fashion. However, moderate consumption was associated with a lower risk of SDM among perimenopausal women. No significant association was found among men and postmenopausal women.

Several constituents of legumes could contribute to the paradoxical effects of legume consumption on mood. The most likely explanation is related to isoflavones, a group of phytoestrogens found almost exclusively in soyabeans and other leguminous plants. As the chemical structure of isoflavones is similar to that of oestrogen, isoflavones can mimic oestrogen's effect on the human body. Consequently, these effects may have positive results on some systems and negative results, similar to hormonal treatment effects, on women's physical health and mental well-being ${ }^{(25)}$. 
Table 1 Sociodemographic and behavioural characteristics by gender and status of severe depressed mood in 4869 adults aged $25-74$ years, NHEFS, $1971-1982^{*}, t, \neq, \S$

\begin{tabular}{|c|c|c|c|c|c|c|c|c|}
\hline \multirow[b]{3}{*}{ Characteristics of participants } & \multicolumn{4}{|c|}{ Men } & \multicolumn{4}{|c|}{ Women } \\
\hline & \multicolumn{2}{|c|}{$\begin{array}{l}\text { Without SDM } \\
\quad(n 1800)\end{array}$} & \multicolumn{2}{|c|}{$\begin{array}{l}\text { With SDM } \\
\quad(n 236)\end{array}$} & \multicolumn{2}{|c|}{$\begin{array}{l}\text { Without SDM } \\
\qquad(n 2307)\end{array}$} & \multicolumn{2}{|c|}{$\begin{array}{l}\text { With SDM } \\
\text { (n 526) }\end{array}$} \\
\hline & $\%$ or mean & SE & $\%$ or mean & SE & $\%$ or mean & SE & $\%$ or mean & SE \\
\hline Follow-up year, mean & $10 \cdot 58$ & $0 \cdot 12$ & $10 \cdot 78 \|$ & $0 \cdot 12$ & $10 \cdot 61$ & $0 \cdot 12$ & $10 \cdot 59$ & $0 \cdot 15$ \\
\hline CES-D score, mean ${ }^{\star \star}$ & $12 \cdot 42$ & $0 \cdot 11$ & $24 \cdot 90 \|$ & 0.65 & $13 \cdot 13$ & $0 \cdot 12$ & $26 \cdot 18 \|$ & 0.43 \\
\hline \multicolumn{9}{|l|}{ Sociodemographic characteristics } \\
\hline Age (years), mean & $43 \cdot 52$ & $0 \cdot 36$ & $43 \cdot 88$ & 0.65 & $43 \cdot 85$ & $0 \cdot 38$ & $44 \cdot 62$ & 0.75 \\
\hline Non-Hispanic White American & $92 \cdot 45$ & 0.90 & $92 \cdot 61$ & $1 \cdot 78$ & $90 \cdot 86$ & 0.93 & 92.98 & 0.96 \\
\hline Living below the poverty line & $19 \cdot 32$ & $1 \cdot 36$ & $27 \cdot 21^{\oplus}$ & $3 \cdot 84$ & $23 \cdot 65$ & $1 \cdot 14$ & $32 \cdot 21 \|$ & $2 \cdot 70$ \\
\hline Centre city of metropolitan & $29 \cdot 21$ & $1 \cdot 89$ & $30 \cdot 13$ & $3 \cdot 47$ & $30 \cdot 33$ & $1 \cdot 74$ & $26 \cdot 62$ & $2 \cdot 48$ \\
\hline Farmer & $4 \cdot 42$ & $0 \cdot 71$ & $3 \cdot 20$ & $0 \cdot 87$ & 3.95 & $0 \cdot 78$ & $6 \cdot 11$ & $1 \cdot 89$ \\
\hline Some years of high school or below & $32 \cdot 06$ & $1 \cdot 64$ & $47 \cdot 33 \|$ & $4 \cdot 06$ & $27 \cdot 52$ & $1 \cdot 23$ & $39 \cdot 67 \|$ & 3.08 \\
\hline Married & $90 \cdot 50$ & $0 \cdot 87$ & $83 \cdot 21 \|$ & $2 \cdot 51$ & $77 \cdot 47$ & 1.09 & $75 \cdot 87$ & $2 \cdot 33$ \\
\hline \multicolumn{9}{|l|}{ Behavioural and dietary characteristics } \\
\hline $\begin{array}{l}\text { Dietary intake of calories, } \mathrm{kJ} / 24 \mathrm{~h} \text {, mean } \\
(\mathrm{kcal} / 24 \mathrm{~h})\end{array}$ & $10193(2435)$ & 159 & $9892(2363)$ & 356 & $6597(1576)$ & 92 & 6199 (1481) & 226 \\
\hline SFA, g/24h, mean & $3 \cdot 81$ & 0.07 & $3 \cdot 47$ & $0 \cdot 15$ & $2 \cdot 37$ & 0.04 & $2 \cdot 179$ & 0.08 \\
\hline Dietary cholesterol intake, $\mathrm{g} / 24 \mathrm{~h}$, mean & $48 \cdot 76$ & $0 \cdot 88$ & $50 \cdot 04$ & $0 \cdot 30$ & $31 \cdot 82$ & 0.63 & $28 \cdot 86$ & $1 \cdot 27$ \\
\hline Presence of any food allergy & $6 \cdot 37$ & 0.66 & $7 \cdot 49$ & $1 \cdot 83$ & $10 \cdot 03$ & $0 \cdot 66$ & $7 \cdot 49$ & $1 \cdot 83$ \\
\hline Cigarette smoking, $100+/$ lifetime ${ }^{\star *}$ & $77 \cdot 90$ & $1 \cdot 38$ & $84 \cdot 14$ & $2 \cdot 35$ & $54 \cdot 44$ & $1 \cdot 45$ & $64 \cdot 22 \|$ & 2.56 \\
\hline Alcohol drinker, heavy & $26 \cdot 05$ & $1 \cdot 61$ & $25 \cdot 03$ & $3 \cdot 24$ & $11 \cdot 33$ & 1.05 & $9 \cdot 94$ & $1 \cdot 76$ \\
\hline Taking vitamin supplement & $20 \cdot 74$ & $1 \cdot 42$ & $19 \cdot 69$ & $3 \cdot 19$ & $26 \cdot 25$ & $1 \cdot 33$ & $28 \cdot 32$ & $2 \cdot 83$ \\
\hline General health status (self-evaluated), poor & $15 \cdot 35$ & $1 \cdot 25$ & $41 \cdot 71 \|$ & $4 \cdot 75$ & $11 \cdot 65$ & $1 \cdot 02$ & $36 \cdot 21 \|$ & 3.04 \\
\hline $\mathrm{BMI} \geq 30 \mathrm{~kg} / \mathrm{m}^{2}$ & $12 \cdot 72$ & 0.96 & $14 \cdot 16$ & $3 \cdot 44$ & $14 \cdot 25$ & 1.09 & $21 \cdot 33 \|$ & $2 \cdot 14$ \\
\hline Total serum cholesterol, $\mathrm{mg} / \mathrm{dL}$, mean & $218 \cdot 26$ & $1 \cdot 54$ & $229 \cdot 249$ & $4 \cdot 76$ & $218 \cdot 22$ & $1 \cdot 49$ & 223.03 & $3 \cdot 15$ \\
\hline \multicolumn{9}{|l|}{ History of medical illness ${ }^{\star \star}$} \\
\hline Cancer, yes & $1 \cdot 45$ & 0.40 & 0.29 ब & 0.29 & $1 \cdot 66$ & 0.41 & $2 \cdot 50,0 \cdot 70$ & $0 \cdot 70$ \\
\hline Diabetes, yes & 1.63 & 0.33 & $1 \cdot 83$ & 0.84 & $2 \cdot 08$ & 0.46 & $1 \cdot 86,0 \cdot 80$ & 0.80 \\
\hline
\end{tabular}

${ }^{*}$ CES-D, Center for Epidemiologic Studies Depression Scale; NHEFS, National Health and Nutrition Examination Survey I Epidemiologic Follow-Up Study; SDM, severe depressed mood.

+Values were calculated using the 1971-1975 survey sampling weights and reported as percentage, unless otherwise indicated

$\ddagger$ Characteristics were assessed at the baseline survey (1971-1975), unless otherwise indicated.

$\S$ The SDM was defined as CES-D score $\geq 22$ and/or taking anti-depression medicine.

$\|$-The $P$-values were generated from Cochran-Mantel-Haenszel test for categorical variables and $t$-test for continuous variables. $\| P$-value $<0 \cdot 01$.

- $P$-value $<0.05$

**The characteristics were assessed at the follow-up survey conducted in 1982 or later.

During menopause, oestrogen level fluctuates naturally. Introducing exogenous hormones from hormonal treatment or plant oestrogens may cause certain anti-nature effects and disturb the biological as well as psychological cycle among women. The gender-dependent association may also relate to the large interindividual variations in the metabolism of isoflavones among men ${ }^{(26)}$. Recent data suggest that the mechanism of action and effectiveness of soya in hormone-dependent studies is largely dependent upon equal production from daidzein, and only about $30 \%$ of men are able to produce equol ${ }^{(27)}$. Although the proportion of frequent legume consumers was higher among men than among women, the proportion of men with SDM was relatively low, indicating that contribution from legume consumption, if any, to the development of depressed mood was much smaller among men than among women. Detecting an association may require more participants from men than from women. Thus, the current study may lack statistical power to detect the association among men.

The second explanation might relate to the paradoxical impact of legume on iron metabolism. Legumes, such as lima beans and green peas, are good sources of iron. However, the bioavailability of iron contained in legumes is very low, as the phytate and some of its degradation products are well-known inhibitors of absorption of essential dietary minerals, especially non-haem iron and Zinc ${ }^{(28)}$. High prevalence and severity of iron deficiency has been observed among populations subsisting predominantly on cereal and legume diets ${ }^{(29)}$, and alteration in iron metabolism has been suggested as a potential pathological marker in patients with manifest depres$\operatorname{sion}^{(30)}$. The anti-nutritional factors from legumes may also lower the nutritional value of a food by lowering the digestibility or bioavailability of other nutrients, such as vitamin B complex, copper and magnesium, which have been linked with the severity of depression among patients $^{(31-33)}$.

Our observation of no association between legume consumption and SDM among men and postmenopausal women indirectly implied that isoflavones and iron metabolism may be the key contributors to the effectmodified association observed by the current study. After menopause, hormone levels of oestrogen and progesterone 
Table 2 Sociodemographic and behavioural characteristics at baseline by gender and the level of legume consumption in 4869 adults aged $25-74$ years, NHEFS, 1971-1982* $,+, \ddagger, \S$

\begin{tabular}{|c|c|c|c|c|c|c|c|c|c|c|c|c|}
\hline \multirow[b]{3}{*}{ Characteristics of participants } & \multicolumn{6}{|c|}{ Men } & \multicolumn{6}{|c|}{ Women } \\
\hline & \multicolumn{2}{|c|}{$<1 /$ week II (n 675) } & \multicolumn{2}{|c|}{ 1-2/week (n 780) } & \multicolumn{2}{|c|}{3 or $+/$ week $(n 581)$} & \multicolumn{2}{|c|}{$<1 /$ week II $(n$ 1280) } & \multicolumn{2}{|c|}{ 1-2/week ( $n$ 1023) } & \multicolumn{2}{|c|}{3 or $+/$ week $(n 530)$} \\
\hline & $\%$ & SE & $\%$ & SE & $\%$ & SE & $\%$ & SE & $\%$ & SE & $\%$ & SE \\
\hline Follow-up year, mean & $10 \cdot 65$ & $0 \cdot 13$ & $10 \cdot 61$ & $0 \cdot 13$ & $10 \cdot 54$ & $0 \cdot 13$ & $10 \cdot 63$ & $0 \cdot 12$ & $10 \cdot 63$ & $0 \cdot 13$ & $10 \cdot 51$ & $0 \cdot 15$ \\
\hline CES-D score, meantt & $13 \cdot 62$ & $0 \cdot 31$ & $14 \cdot 07$ & 0.33 & 13.92 & $0 \cdot 30$ & $15 \cdot 24$ & $0 \cdot 29$ & $15 \cdot 38$ & $0 \cdot 27$ & $15 \cdot 85$ & 0.46 \\
\hline With severe depressive symptomstt & 11.96 & 1.55 & $12 \cdot 77$ & $1 \cdot 79$ & $9 \cdot 81$ & $1 \cdot 44$ & $17 \cdot 09$ & $1 \cdot 33$ & $16 \cdot 04$ & $1 \cdot 54$ & $23 \cdot 31^{\star \star}$ & $2 \cdot 29$ \\
\hline \multicolumn{13}{|l|}{ Sociodemographic characteristics } \\
\hline Age (years), mean & $44 \cdot 60$ & 0.56 & $42 \cdot 99$ & 0.52 & $43.08 \%$ & 0.56 & $45 \cdot 42$ & $0 \cdot 41$ & $42 \cdot 40$ & 0.59 & $43.00 \%$ & 0.72 \\
\hline Race, White American & $92 \cdot 38$ & $1 \cdot 59$ & $93 \cdot 15$ & $1 \cdot 16$ & $91 \cdot 61$ & $1 \cdot 45$ & $92 \cdot 60$ & 0.90 & $89 \cdot 19$ & $1 \cdot 52$ & $91 \cdot 43$ & $1 \cdot 28$ \\
\hline Living below the poverty line & $18 \cdot 15$ & $1 \cdot 81$ & $18 \cdot 97$ & $1 \cdot 79$ & $24 \cdot 65$ & $2 \cdot 71$ & $18 \cdot 68$ & $1 \cdot 52$ & $29 \cdot 71$ ब & $1 \cdot 83$ & $35 \cdot 12^{\star \star}$ & 3.09 \\
\hline Centre city of metropolitan & $33 \cdot 46$ & $2 \cdot 80$ & $26 \cdot 00$ & $2 \cdot 20$ & $28 \cdot 88^{\circ}$ & $2 \cdot 66$ & $29 \cdot 91$ & $1 \cdot 87$ & $29 \cdot 67$ & $2 \cdot 50$ & 28.98 & $2 \cdot 19$ \\
\hline Farmer & 3.88 & $0 \cdot 89$ & $4 \cdot 96$ & 1.06 & 3.79 & 0.99 & $3 \cdot 72$ & 0.59 & $4 \cdot 88^{\circ}$ & $1 \cdot 09$ & 4.99 & 1.53 \\
\hline Some years of high school or below & $33 \cdot 02$ & $2 \cdot 62$ & $31 \cdot 27$ & $2 \cdot 45$ & $38 \cdot 55^{\star \star}$ & $2 \cdot 58$ & $27 \cdot 31$ & $1 \cdot 52$ & $29 \cdot 31$ & $1 \cdot 81$ & $37 \cdot 52$ & $2 \cdot 68$ \\
\hline Married & $87 \cdot 40$ & $1 \cdot 69$ & $91 \cdot 00$ & $1 \cdot 12$ & $90 \cdot 51$ & $1 \cdot 32$ & $75 \cdot 58$ & $1 \cdot 73$ & $78 \cdot 53$ & $1 \cdot 80$ & $79 \cdot 18$ & $2 \cdot 05$ \\
\hline \multicolumn{13}{|l|}{ Behavioural and dietary characteristics } \\
\hline Dietary calories, $\mathrm{kJ} / 24 \mathrm{~h}$, mean $(\mathrm{kcal} / 24 \mathrm{~h})$ & $10185(2433)$ & 272 & $9988(2386)$ & 197 & $10365(2476)$ & 201 & $6472(1546)$ & 138 & $6388(1526)$ & 30 & $6974(1666)$ & 268 \\
\hline SFA, g/24h, mean & 3.55 & $0 \cdot 1$ & 3.79 & $0 \cdot 10$ & $4 \cdot 02^{\star \star}$ & 0.97 & $2 \cdot 26$ & 0.06 & $2 \cdot 28$ & 0.05 & 2.55 & 0.08 \\
\hline Cholesterol intake, $\mathrm{g} / 24 \mathrm{~h}$, mean & $47 \cdot 87$ & $1 \cdot 41$ & $48 \cdot 58$ & $1 \cdot 33$ & $50 \cdot 66$ & $1 \cdot 57$ & 31.90 & $0 \cdot 88$ & $29 \cdot 76$ & $1 \cdot 01$ & $32 \cdot 68$ & 1.96 \\
\hline Presence of any food allergy & $6 \cdot 88$ & $1 \cdot 23$ & $6 \cdot 70$ & $1 \cdot 00$ & $5 \cdot 73$ & $0 \cdot 79$ & $10 \cdot 60$ & $1 \cdot 18$ & $8 \cdot 87$ & $1 \cdot 29$ & $9 \cdot 65$ & 1.64 \\
\hline Cigarette smoking, 100/lifetimett & $76 \cdot 39$ & $2 \cdot 12$ & $79 \cdot 70$ & $2 \cdot 28$ & $79 \cdot 89$ & 1.95 & $55 \cdot 99$ & $1 \cdot 89$ & $55 \cdot 64$ & 1.92 & $57 \cdot 87$ & 3.34 \\
\hline Alcohol drinker, heavy & $26 \cdot 93$ & $2 \cdot 50$ & $24 \cdot 82$ & $2 \cdot 72$ & $26 \cdot 26$ & $2 \cdot 33$ & $12 \cdot 89$ & 1.55 & 8.00 & $1 \cdot 13$ & $12 \cdot 13$ & $1 \cdot 72$ \\
\hline Taking vitamin supplement & $19 \cdot 63$ & $2 \cdot 14$ & $20 \cdot 85$ & $2 \cdot 14$ & $21 \cdot 50$ & $2 \cdot 78$ & $26 \cdot 43$ & $1 \cdot 70$ & $25 \cdot 01$ & 1.90 & $30 \cdot 53$ & $2 \cdot 68$ \\
\hline General health status, poortt & $18 \cdot 01$ & $1 \cdot 86$ & $17 \cdot 53$ & 1.98 & $20 \cdot 23$ & 1.92 & $16 \cdot 05$ & $1 \cdot 40$ & $15 \cdot 52$ & 1.49 & $16 \cdot 91$ & $2 \cdot 34$ \\
\hline Obese, $\left(\mathrm{BMI} \geq 30 \mathrm{~kg} / \mathrm{m}^{2}\right)$ & $13 \cdot 41$ & $1 \cdot 60$ & $14 \cdot 68$ & $1 \cdot 74$ & $9 \cdot 68$ & $1 \cdot 50$ & $15 \cdot 56$ & $1 \cdot 37$ & $17 \cdot 38$ & 1.99 & $11 \cdot 45$ & $2 \cdot 02$ \\
\hline Total serum cholesterol, $\mathrm{mg} / \mathrm{dL}$, mean & $220 \cdot 62$ & $2 \cdot 17$ & $221 \cdot 32$ & $2 \cdot 27$ & $215 \cdot 68$ & $2 \cdot 86$ & $222 \cdot 72$ & $2 \cdot 08$ & $216 \cdot 36$ & $2 \cdot 11$ & 213.89 & $2 \cdot 12$ \\
\hline \multicolumn{13}{|l|}{ Lifetime history of medical illnesstt } \\
\hline Cancer, yes & $1 \cdot 29$ & $0 \cdot 65$ & $1 \cdot 25$ & 0.51 & $1 \cdot 44$ & 0.64 & 1.99 & 0.54 & $1 \cdot 15$ & 0.54 & $2 \cdot 63$ & 1.00 \\
\hline Diabetes, yes & $2 \cdot 05$ & 0.54 & 1.00 & 0.38 & $2 \cdot 08$ & $0 \cdot 67$ & 1.97 & 0.46 & $2 \cdot 29$ & 0.83 & $1 \cdot 72$ & 0.61 \\
\hline
\end{tabular}

*CES-D, Center for Epidemiologic Studies Depression Scale; NHEFS, National Health and Nutrition Examination Survey I Epidemiologic Follow-Up Study; SDM, severe depressed mood.

+Values were calculated using the 1971-1975 survey sampling weights and reported as percentage, unless otherwise indicated.

$¥$ Characteristics were assessed at the baseline survey (1971-1975), unless otherwise indicated.

§The SDM was defined as CES-D score $\geq 22$ and/or taking anti-depression medicine.

IIReferent group for each gender, pairwise comparisons were made between referent group and other two groups within the same gender.

T- ${ }^{* \star}$ The $P$-values were generated from Cochran-Mantel-Haenszel test for categorical variables and $t$ test for continuous variables. ${ }^{\uparrow} P$-value $<0 \cdot 05$. ${ }^{* \star} P$-value $<0 \cdot 017(0 \cdot 05 / 3$, Bonferroni-adjusted test), and reference level was the group with legumes consumed less than once a week.

$\mathrm{t}$ The characteristics were assessed at the follow-up survey conducted in 1982 or later. 
Table 3 Risk ratios for SDM associated with legume consumption in 4869 adults aged 25-74 years, NHEFS, 1971-1982*

\begin{tabular}{|c|c|c|c|c|c|c|c|}
\hline & \multirow[b]{2}{*}{$\begin{array}{l}\text { Unweighted } \\
\text { sample size }\end{array}$} & \multicolumn{2}{|c|}{ Subjects with SDM +} & \multicolumn{2}{|c|}{ Unadjusted RR } & \multicolumn{2}{|c|}{ Adjusted RR $\ddagger$} \\
\hline & & Weighted \% & $\%$ SE & $\begin{array}{c}\text { Point } \\
\text { estimate }\end{array}$ & $95 \% \mathrm{Cl}$ & $\begin{array}{c}\text { Point } \\
\text { estimate }\end{array}$ & $95 \% \mathrm{Cl}$ \\
\hline \multicolumn{8}{|l|}{ Men ( $\geq 25$ years, $n$ 2036) } \\
\hline$<1$ time/week & 675 & $10 \cdot 31$ & 1.55 & 1.00 & (reference) & $1 \cdot 00$ & (reference) \\
\hline 1-2 times/week & 780 & $12 \cdot 77$ & $1 \cdot 79$ & $1 \cdot 08$ & $0.69,1.68$ & $1 \cdot 04$ & $0.65,1.67$ \\
\hline 3 times or more/week & 581 & $8 \cdot 28$ & $1 \cdot 44$ & 0.80 & $0 \cdot 50,1 \cdot 28$ & 0.73 & $0 \cdot 45,1 \cdot 18$ \\
\hline$P$ for linear trend & & & & $0 \cdot 3532$ & & $0 \cdot 1966$ & \\
\hline \multicolumn{8}{|c|}{ Premenopausal women§ (25-43 years, $n$ 1778) } \\
\hline$<1$ time/week & 727 & $14 \cdot 24$ & $1 \cdot 45$ & $1 \cdot 00$ & (reference) & $1 \cdot 00$ & (reference) \\
\hline 1-2 times/week & 701 & $17 \cdot 82$ & $1 \cdot 91$ & $1 \cdot 31$ & $0.97,1 \cdot 76$ & $1 \cdot 23$ & $0.91,1.68$ \\
\hline 3 times or more/week & 350 & $24 \cdot 38$ & $2 \cdot 97$ & 1.94 & $1 \cdot 28,2 \cdot 95$ & $1 \cdot 76$ & $1 \cdot 13,2 \cdot 74$ \\
\hline$P$ for linear trend & & & & 0.0019 & & $0 \cdot 0122$ & \\
\hline \multicolumn{8}{|c|}{ Perimenopausal women $\S(44-55$ years, $n$ 454) } \\
\hline$<1$ time/week & 230 & $19 \cdot 32$ & $2 \cdot 54$ & 1.00 & (reference) & $1 \cdot 00$ & (reference) \\
\hline 1-2 times/week & 143 & $11 \cdot 38$ & $3 \cdot 88$ & 0.54 & $0.28,1 \cdot 02$ & 0.52 & $0.27,1.00$ \\
\hline 3 times or more/week & 81 & $20 \cdot 51$ & $4 \cdot 31$ & $1 \cdot 08$ & $0.60,1.94$ & $1 \cdot 29$ & $0.63,2.66$ \\
\hline$P$ for quadratic trend & & & & 0.0517 & & 0.0233 & \\
\hline \multicolumn{8}{|c|}{ Postmenopausal women§ (55+ years, $n$ 601) } \\
\hline$<1$ time/week & 323 & $19 \cdot 76$ & $2 \cdot 99$ & 1.00 & (reference) & $1 \cdot 00$ & (reference) \\
\hline 1-2 times/week & 179 & $16 \cdot 52$ & $3 \cdot 91$ & $0 \cdot 80$ & $0.43,1.48$ & $0 \cdot 65$ & $0.28,1.51$ \\
\hline 3 times or more/week & 99 & $24 \cdot 26$ & $6 \cdot 69$ & $1 \cdot 30$ & $0.54,3.09$ & $1 \cdot 55$ & $0.62,3.85$ \\
\hline$P$ for quadratic trend & & & & $0 \cdot 2671$ & & $0 \cdot 1244$ & \\
\hline
\end{tabular}

*NHEFS, National Health and Nutrition Examination Survey I Epidemiologic Follow-Up Study; RR, relative risk; SDM, severe depressed mood.

tThe sample sizes were presented as unweighted, but the proportions were estimated as weighted using appropriate weighting and nesting variables.

$\ddagger$ The variables of socio-economic status included race/ethnicity, education attainment, family income level, marital status, types of residence area, occupations and employment status assessed at the baseline. Other covariates included the BMI, alcohol drinking, cigarette smoking, serum total cholesterol, total dietary energy intake, SFA intake, frequency of eating fruit and vegetables and presence of food allergy assessed at the baseline survey and self-evaluate health status and the history of major physical diseases (cancer, diabetes, stroke and heart attack) assessed at the follow-up survey.

$\S$ The status of menopause was assessed based on the age at the baseline interview and the answer to the question 'Have they (periods) entirely stopped?'.

are much lower and less fluctuant, and women's requirement for iron is also substantially reduced, almost reaching the same level as for men. However, other factors may also play a role to the mixed effects from legumes. The Brain Amine Theory speculates that a shortage of amino acids, particularly the essential amino acids, can impact negatively on mental health ${ }^{(34)}$. Legumes do not have an ideal balance of amino acids. If the needed amino acid is not available, the level of particular neurotransmitters in the brain will fall, and brain functioning and mood will be affected. Vegetarian and semivegetarian women generally consume legumes as their main source of proteins. Whether the current findings are relevant to previous studies ${ }^{(35-38)}$, showing that vegetarians had a poor mental health, needs to be further examined. Numerous studies have linked low serum cholesterol and depressed mood ${ }^{(39,40)}$, and total serum cholesterol was significantly lower among women who ate legumes frequently. Although we included the serum total cholesterol as a covariate, the residual confounding from cholesterol remained possible ${ }^{(41)}$. Further studies are also needed to examine the potential effects from high intake of protein from legumes. We observed that the proportions of women living below the poverty line significantly and gradually increased as frequency of legume consumption increased. Therefore, it was likely that residual confounding from family income contributed to the association observed among women. However, these residual confounding effects, if any, should not differ by the menopausal status.

Limitations of this investigation include possible bias arising from loss to follow-up and missing data on baseline survey. Most persons who died were lost to follow-up or excluded due to missing covariates, and were of low SES or the elderly. These individuals were more vulnerable to detrimental effects from the risk factors ${ }^{(1-19)}$. Use of a single FFQ survey may introduce errors due to daily or seasonal variability of dietary pattern. Dietary practices may also have changed during the 10-year follow-up period. The physiological effects of different legumes vary significantly due to the variability from polysaccharide composition, protein makeup and phytochemical contents. Furthermore, most grain legumes are consumed after simple processing, as vegetables, salads, soups, mashed and cooked seeds. The current study using FFQ was not able to distinguish between the individual characteristics of this heterogeneous family of foods, so misclassification was unavoidable. All these biases, if any, should lead to a dilution of the RR towards unity. In addition, the CES-D scale is neither accurate in measuring past episodes ${ }^{(42)}$ nor in identifying individuals with major mood disorder. Major mood disorder has been previously shown to be most closely related to nutrition intervention in clinical trials ${ }^{(43)}$. Only a portion of the NHANES I participants were invited for a more detailed medical examination at baseline, preventing us 
from excluding the individuals with a history of psychiatric disorders. However, our study had several strengths that are noteworthy. To the best of our knowledge, this is the first study showing a modified effect of legume consumption on depressed mood. Most of the earlier studies have focused on specific components of legumes, such as protein, fibre and phytochemicals, or limited with animal models. However, studies focusing on specific nutrients may fail to consider the biochemical complexity and possibility of nutrient interactions in food items. Moreover, animal models generally fail to reliably predict human response in theory and practice ${ }^{(44)}$. The data of food consumption were collected four decades ago, when the consumption of processed food with enriched isoflovones or fortified iron was relatively low ${ }^{(45)}$. Therefore, the confounding effects from food additives, if any, might be at a low level, and the effects observed are much natural.

\section{Conclusion}

In conclusion, our investigation found an effect-modified association between legume consumption and the risk of SDM. Among women, this association was menopause status-dependent. More consumption of legumes was associated with an increased risk of SDM for premenopausal women. However, moderate consumption showed protective effects among perimenopausal women. Additional studies are clearly warranted to examine this nonmonotonic association. Fundamental knowledge of the nutritional behaviours of legumes in complex food system is critically required to propose ingredients and adaptations to formulations for appetising foods, and make health recommendations for physical health and mental well-being as well.

\section{Acknowledgements}

The current study has been conducted with support from the academic units with which the authors are affiliated. The NHANES I Epidemiologic Follow-up Study has been developed and funded by the following agencies: National Center for Health Statistics; National Institute on Aging; National Cancer Institute; National Center for Chronic Disease Prevention and Health Promotion; National Institute of Child Health and Human Development; National Heart, Lung, and Blood Institute; National Institute on Alcohol Abuse and Alcoholism; National Institute of Mental Health; National Institute of Diabetes and Digestive and Kidney Diseases; National Institute of Arthritis and Musculoskeletal and Skin Diseases; National Institute of Allergy and Infectious Diseases; National Institute of Neurological and Communicative Disorders and Stroke; and the US Department of Agriculture. The fieldwork was conducted by Westat Inc. under contract to the National Center for Health
Statistics. All authors contributed to the study design; J.Z. supervised the study; Y.L. and Q.D. obtained the data, performed data analysis and drafted the manuscript, and all authors participated in critically revising the manuscript for important intellectual content. None of the authors had any financial or personal interest, including advisory board affiliations, in any company or organisation within the food or pharmaceutical industries.

\section{References}

1. Krauss RM, Eckel RH, Howard B et al. (2000) AHA dietary guidelines: revision 2000: a statement for healthcare professionals from the Nutrition Committee of the American Heart Association. Stroke 31, 2751-2766.

2. Glade MJ (1999) Food, nutrition, and the prevention of cancer: a global perspective. American Institute for Cancer Research/World Cancer Research Fund, American Institute for Cancer Research, 1997. Nutrition 15, 523-526.

3. Venn BJ \& Mann JI (2004) Cereal grains, legumes and diabetes. Eur J Clin Nutr 58, 1443-1461.

4. Bazzano LA, He J, Ogden LG et al. (2001) Legume consumption and risk of coronary heart disease in US men and women: NHANES I Epidemiologic Follow-up Study. Arch Intern Med 161, 2573-2578.

5. Azadbakht L, Kimiagar M, Mehrabi Y et al. (2007) Soy inclusion in the diet improves features of the metabolic syndrome: a randomized crossover study in postmenopausal women. Am J Clin Nutr 85, 735-741.

6. Flight I \& Clifton P (2006) Cereal grains and legumes in the prevention of coronary heart disease and stroke: a review of the literature. Eur J Clin Nutr 60, 1145-1159.

7. The Scientific Committee of the AEP (2001) Benefits for human health - a scientific consensus. http://www.grain legumes.com/aep (accessed February 2007).

8. The Scientific Committee of the AEP (2005) AEP expert appraisal for legume research beyond 2005. http:// www.grainlegumes.com/aep (accessed January 2007).

9. Farnsworth NR, Krause EC, Bolton JL et al. (2008) The University of Illinois at Chicago/National Institutes of Health Center for Botanical Dietary Supplements Research for Women's Health: from plant to clinical use. Am J Clin Nutr 87, 504S-508S.

10. Patisaul HB, Blum A, Luskin JR et al. (2005) Dietary soy supplements produce opposite effects on anxiety in intact male and female rats in the elevated plus-maze. Behav Neurosci 119, 587-594.

11. Patisaul HB (2005) Phytoestrogen action in the adult and developing brain. J Neuroendocrinol 17, 57-64.

12. Patisaul HB, Luskin JR \& Wilson ME (2004) A soy supplement and tamoxifen inhibit sexual behavior in female rats. Horm Behav 45, 270-277.

13. Patisaul HB, Dindo M, Whitten PL et al. (2001) Soy isoflavone supplements antagonize reproductive behavior and estrogen receptor alpha- and beta-dependent gene expression in the brain. Endocrinology 142, 2946-2952.

14. Hunt JR (2002) Moving toward a plant-based diet: are iron and zinc at risk? Nutr Rev 60, 127-134.

15. Ma G, Li Y, Jin Y et al. (2007) Phytate intake and molar ratios of phytate to zinc, iron and calcium in the diets of people in China. Eur J Clin Nutr 61, 368-374.

16. Zhang J, Li Y \& Torres ME (2005) How does a suicide attempter eat differently from others? Comparison of macronutrient intakes. Nutrition 21, 711-717.

17. Ingram DD \& Makuc DM (1994) Statistical issues in analyzing the NHANES I Epidemiologic Followup Study. 
Series 2: data evaluation and methods research. Vital Health Stat, 2, 1-30.

18. Madans JH, Cox CS, Kleinman JC et al. (1986) 10 years after NHANES I: mortality experience at initial followup, 1982-84. Public Health Rep 101, 474-481.

19. Madans JH, Kleinman JC, Cox CS et al. (1986) 10 years after NHANES I: report of initial followup, 1982-84. Public Health Rep 101, 465-473.

20. National Center for Health Statistics (1997) Laboratory Procedures Used in the Third National Health and Nutrition Examination Survey (NHANES III, 1988-94). Hyattsville, MD: US Department of Health and Human Service, Public Health Service, Center for Disease Control and Prevention.

21. US Department of Health and Human Services, Centers for Disease Control National Center for Health Statistics, US Government (1991) Public Use Data Tape Documentation, Dietary Frequency and Adequacy, Ages 1-74, Tape Number 4701, National Health and Nutrition Examination Survey, 1971-75. Hyattsville, MD: US Government.

22. Weissman MM, Sholomskas D, Pottenger M et al. (1977) Assessing depressive symptoms in five psychiatric populations: a validation study. Am J Epidemiol 106, 203-214.

23. Zhang J, McKeown RE, Hussey JR et al. (2005) Gender differences in risk factors for attempted suicide among young adults: findings from the Third National Health and Nutrition Examination Survey. Ann Epidemiol 15, 167-174.

24. Li Y, Zhang J \& McKeown RE (2007) Cross-sectional assessment of diet quality in individuals with a life-time history of attempted suicide. Psychiatry Res 153, 111-112.

25. Moutsatsou P (2007) The spectrum of phytoestrogens in nature: our knowledge is expanding. Hormones (Athens) 6, 173-193.

26. Rowland I, Faughnan M, Hoey L et al. (2003) Bioavailability of phyto-oestrogens. Br J Nutr 89, Suppl 1, S45-S58.

27. Bryant M, Cassidy A, Hill C et al. (2005) Effect of consumption of soy isoflavones on behavioural, somatic and affective symptoms in women with premenstrual syndrome. Br J Nutr 93, 731-739.

28. Lynch SR, Beard JL, Dassenko SA et al. (1984) Iron absorption from legumes in humans. Am J Clin Nutr $\mathbf{4 0}$, 42-47.

29. Hurrell RF (2004) Phytic acid degradation as a means of improving iron absorption. Int J Vitam Nutr Res 74, 445-452.

30. Li Y, Dai Q, Torres ME et al. (2007) Gender-specific association between iron status and the history of attempted suicide: Implications for gender paradox of suicide behaviors. Prog Neuropsychopharmacol Biol Psychiatry 31, 1429-1435.

31. Zieba A, Kata R, Dudek D et al. (2000) Serum trace elements in animal models and human depression: Part III.
Magnesium. Relationship with copper. Hum Psychopharmacol 15, 631-635.

32. Poleszak E (2007) Modulation of antidepressant-like activity of magnesium by serotonergic system. J Neural Transm 114, 1129-1134.

33. Kamei K, Tabata O, Muneoka KATS et al. (1998) Electrolytes in erythrocytes of patients with depressive disorders. Psychiatry Clin Neurosci 52, 529-533.

34. Sullivan GM, Mann JJ, Oquendo MA et al. (2006) Low cerebrospinal fluid transthyretin levels in depression: correlations with suicidal ideation and low serotonin function. Biol Psychiatry 60, 500-506.

35. Baines S, Powers J \& Brown WJ (2007) How does the health and well-being of young Australian vegetarian and semi-vegetarian women compare with non-vegetarians? Public Health Nutr 10, 436-442.

36. Larsson CL, Klock KS, Nordrehaug Astrom A et al. (2002) Lifestyle-related characteristics of young low-meat consumers and omnivores in Sweden and Norway. $J$ Adolesc Health 31, 190-198.

37. Neumark-Sztainer D, Story M, Resnick MD et al. (1997) Adolescent vegetarians. A behavioral profile of a schoolbased population in Minnesota. Arch Pediatr Adolesc Med 151, 833-838.

38. Perry CL, Mcguire MT, Neumark-Sztainer D et al. (2001) Characteristics of vegetarian adolescents in a multiethnic urban population. J Adolesc Health 29, 406-416.

39. Garland MR, Hallahan B, McNamara M et al. (2007) Lipids and essential fatty acids in patients presenting with self-harm. Br J Psychiatry 190, 112-117.

40. Zhang J, McKeown RE, Hussey JR et al. (2005) Low HDL cholesterol is associated with suicide attempt among young healthy women: the third National Health and Nutrition Examination Survey. J Affect Disord 89, 25-33.

41. Fewell Z, vey Smith G \& Sterne JAC (2007) The impact of residual and unmeasured confounding in epidemiologic studies: a simulation study. Am J Epidemiol 166, 646-655.

42. Breslau N (1985) Depressive symptoms, major depression, and generalized anxiety: a comparison of self-reports on CES-D and results from diagnostic interviews. Psychiatry Res 15, 219-229.

43. Appleton KM, Hayward RC, Gunnell D et al. (2006) Effects of $n$-3 long-chain polyunsaturated fatty acids on depressed mood: systematic review of published trials. Am J Clin Nutr 84, 1308-1316.

44. Knight A (2007) Systematic reviews of animal experiments demonstrate poor human clinical and toxicological utility. Altern Lab Anim 35, 641-659.

45. Choi MS \& Rhee KC (2006) Production and processing of soybeans and nutrition and safety of isoflavone and other soy products for human health. J Med Food 9, 1-10. 